\title{
SEMINORMAL GRADED RINGS AND WEAKLY NORMAL PROJECTIVE VARIETIES
}

\author{
JOHN V. LEAHY \\ Institute of Theoretical Sciences \\ and \\ Department of Mathematics, University of Oregon \\ Eugene, Oregon 97403 \\ and
} MARIE A. VITULLI

Department of Mathematics, University of Oregon Eugene, Oregon 97403

(Received March 4, 1983)

ABSTRACT. This paper is concerned with the seminormality of reduced graded rings and the weak normality of projective varieties. One motivation for this investigation is the study of the procedure of blowing up a non-weakly normal variety along its conductor ideal.

KEY WORDS AND PHRASES. Seminormal Graded Rings, Normal Proiective Varieties. 1980 MATHEMATICS SUBJECT CLASSIFICATION CODE. $16 A 03$.

\section{INTRODUCTION.}

In this paper we investigate the seminormality of reduced graded rings for the purpose of studying the weak normality of projective varieties and the procedure of blowing up a non-weakly normal variety along its conductor ideal. A commutative ring with identity is seminormal if and only if it contains every element of its total ring of quotients whose square and cube are in the ring. A variety (over an algebraically closed field of characteristic zero) is weakly normal if and only if every globally defined continuous function which is regular outside the singular locus is in fact globally regular. In [1] it is proven that a variety is weakly normal if and only if all of its local rings are seminormal.

In section 2 of this paper the relevant commutative algebra is developed to handle questions of weak normality for projective varieties and in particular to show that the weak normalization of a projective variety is again projective. We have tried to work in as general an algebraic setting as possible, but since we wanted the normalization of a graded ring to again be graded we considered only reduced graded rings (indexed by the integers) having only a finite number of minimal prime ideals. Given an integral extension of graded rings we give an explicit description of the relative seminormalization by adapting a construction of Swan [2]. We then show that the seminormalization of a graded ring is a graded subring of its normalization and that the operations of seminormalization and homogeneous localization commute. 
In section 3 we consider reduced (but not necessarily irreducible) projective varieties defined over an algebraically closed field of characteristic zero. In particular we show that such a variety is weakly normal if and only if its veronese subrings of order $d$ are seminormal for sufficiently large $d$.

2. SEMINORMAL GRADED RINGS.

All rings are assumed to be commutative with identity. A graded ring is a ring $R$ that admits a decomposition (as abelian groups) $R=\oplus_{n \in \mathbb{Z}} R_{n}$ such that $R_{m} R_{n} \subseteq R_{m+n}$ for all $\mathrm{m}, \mathrm{n}$. We let $\mathrm{R}^{\mathrm{h}}$ denote the set of homogeneous elements of $R$. Notice that the identity element of $R$ is homogeneous of degree 0 . In particular $R_{0}$ is a subring of $R$.

If $A$ is a ring we let $\bar{A}$ denote its normalization, i.e. $\bar{A}$ is the integral closure of $A$ in its total ring of quotients $Q(A)$.

(2.0) Suppose that $R$ is a reduced graded ring having only a finite number of minimal primes $\rho_{1}, \cdots, \rho_{\ell}$. Let $R^{i}=R_{/} \rho_{i}, T_{i}=R^{h}-\rho_{i}$ and $S^{i}=T_{i}^{-1} R^{i}$ for $i=1, \cdots, x$. Set $S=S^{1} \times \cdots \times S$. Since the minimal primes of $R$ are homogeneous the quotient rings $R^{i}$ are again graded. Therefore each $S^{i}$ is graded where $s_{n}^{i}=\left\{\alpha / t \mid \alpha \varepsilon\left(R^{i}\right)^{h}, t \in T^{i}\right.$ and $\operatorname{deg}(\alpha)-\operatorname{deg}(t)=n j$. Hence $s=\oplus_{n \varepsilon \mathbb{Z}^{n}} S_{n}^{1} \times \cdots S_{n}^{\chi}$ is a graded ring.

Notice that $R$ is a graded subring of $R^{1} \times \cdots \times R$. Suppose $r \varepsilon R \cap\left(R_{n}^{1} \times \cdots \times R_{n}^{\ell}\right)$. Then there exist homogeneous elements $r_{i}$ of degree $n$ and $s_{i} \varepsilon \rho_{i}$ such that $r=r_{i}+s_{i}$ for $i=1, \cdots, l$. For each $i$ let $s_{i}(n)$ denote the homogeneous component of $s_{i}$ of degree $n$. Replacing $r_{i}$ by $r_{i}+s_{i}(n)$ and $s_{i}$ by $s_{i}-s_{i}(n)$ we may assume that $s_{i}(n)=0$ for all $i$. Comparing homogeneous components of iegree $n$ we see that $r_{i}=r_{1}$ for all $i$ and consequently $s_{i}=s_{1}$ for all i. Thus $s_{1} \varepsilon \bigcap_{i}=0$ and $r=r_{1}$ is homogeneous of degree $n$.

Now $R^{i}$ is a graded subring of $S^{i}(i=1, \cdots, l)$ so that $R^{1} \times \ldots \times R$ is a graded subring of $S=S^{1} x_{\cdots} \ldots S^{\ell}$. Consequently $R$ is a graded subring of $S$.

LEMIA 2.1. Let $R$ be a reduced graded ring having only a finite number of minimal primes. With notation as above, the normalization $\bar{R}$ of $R$ is a graded subring of $S$. If $R_{n}=0$ for all negative integers $n$ then $\bar{R}_{n}=0$ for all negative integers $\mathrm{m}$.

PROOF. We know that $\bar{R} \simeq \bar{R}^{1} \times \ldots \bar{R}^{l}$ with notation as in (2.0). By the domain case ([e], vol. 2, Thm. 11, p.157) $\overline{R^{i}}$ is a graded subring of $s^{i}$ for each $i$. Thus $\bar{R}$ is a graded subring of $S=S^{1} \times \ldots \times S^{\ell}$. Now assume that $R_{n}=0$ for al1 $\mathrm{n}<0$. Again by the domain case $\left(R^{i}\right)_{n}=0$ for al1 $n<0 \quad(i=1, \cdots, l)$ so that $\bar{R}_{n}=0$ for all $n<0$.

LEITA 2.2. Let $R$ be a reduced graded ring having only a finite number of minimal primes. Assume that there exists a homogeneous R-regular element of positive degree. Let $T$ denote the set of homogeneous R-regular elements of $R$. Then $\bar{R}$ is a graded subring of $T^{-1} R$.

PROOF. Let $\rho_{i}, R^{i}, T_{i}$ be as in (2.0). Let $q_{1}, \cdots, q_{\ell}$ denote the minimal primes of $\bar{R}$ indexed so that $q_{i} \cap_{R}=\rho_{i} \quad(i=1, \cdots, l)$.

Now suppose $\beta \in \bar{R}$. By 2.1 there exist $a_{i}{ }^{r} T_{i}$ such that $a_{i} \beta \varepsilon R+q_{i}$ $(i=1, \cdots, l)$. For each $i$ choose a homogeneous element $b_{i} \varepsilon \cap_{j \neq i} \rho_{j}-\rho_{i} \cdot \operatorname{Re}-$ 
placing $a_{i}$ by $a_{i} b_{i}$ we may assume that $a_{i} \varepsilon \cap_{j \neq i} \rho_{j}-\rho_{i}$ and $a_{i} \beta \varepsilon R$. Let a be a homogeneous R-regular element of positive degree and choose $m$ so that $\operatorname{deg}\left(a_{i}\right)+m>0$ for $i=1, \cdots, l$. Replacing $a_{i}$ by $a_{i} a^{m}$ we may assume that $n_{i}=\operatorname{deg}\left(a_{i}\right)>0$ where $a_{i} \varepsilon \bigcap_{j \neq i} \rho_{j}-\rho_{i}$ and $a_{i} \beta \varepsilon R$ for $i=1, \cdots, l$. $m_{i}$ Let $N=n_{1} \cdots n_{\ell}$ and $l e t m_{i}=N / n_{i}$ for $i=1, \cdots, \ell$. Replacing $a_{i}$ by $a_{i} i$ we may assume that $\operatorname{deg}\left(a_{i}\right)=N>0$ for $i=1, \cdots, \ell$. Then $c=a_{1}+\cdots+a_{\ell}$ is homogeneous and $c \beta r R$. To finish the proof it suffices to see that $c$ is R-regular.

Suppose that $c$ is a zero divisor in $R$ so that $c{ }^{f} \rho_{i}$ for some $i$. Now $c=a_{i}+\sum_{j \neq i} a_{j}$ and $\sum_{j \neq i} a_{j} \varepsilon \rho_{i}$ implies $a_{i} \varepsilon \rho_{i}$, a contradiction. Thus $c$ is R-regular and $\beta \in T^{-1} R$. Hence $\bar{R} \subseteq T^{-1} R$.

One can easily check that $T^{-1} R$ is a graded subring of $S=T_{1}^{-1} R^{1} \times \cdots \times T_{\chi}^{-1} R^{\ell}$. Since $\bar{R}$ is a graded subring of $S$ by 2.1 we know that $\bar{R}$ is a graded subring of $\mathrm{T}^{-1} \mathrm{R}$.

For a graded ring $R=\oplus_{n \in \mathbb{Z}^{R}}$ and a positive integer $d$ we let $R(d)$ denote the subring of $R$ defined by $R(d)=\oplus_{n \in \mathbb{Z}}{ }_{n d}$. Then $R(d)$ is a graded ring where $R(d)=R_{n d}$.

If $R$ is the homogeneous coordinate ring of a projective variety then $R(d)$ is called a Veronese subring of $R$.

COROLLARY 2.3. Let $R$ be a reduced graded ring having only a finite number of minimal primes. Let $\bar{R}$ denote the normalization of $R$. For each positive integer $d, \bar{R}(d)$ is the normalization of $R(d)$.

PROOF. Let $q_{1}, \cdots, q_{\ell}$ denote the minimal primes of $\bar{R}$ and let $\rho_{i}=q_{1} \cap R$ for $i=1, \cdots, l$. By $2.1 \bar{R}$ is a graded ring and hence each $q_{i}$ is a homogeneous ideal. Since $R$ is reduced we know that $\cap_{i=1}^{l} q_{i}=0$. In particular there exist homogeneous elements $\beta_{i} \varepsilon \bar{R}$ such that $q_{i}=\left(0: \beta_{i}\right)$ for $i=1, \cdots, l$.

Suppose $\alpha \varepsilon \bar{R}(d)$ is $\bar{R}(d)$-regular. If $\alpha$ is a zero divisor in $\bar{R}$ then $\alpha \beta_{i}=0$ for some $i$. But $0 \neq \beta_{i}^{d} \varepsilon \bar{R}(d)$ and $\alpha \beta_{i}^{d}=0$, a contradiction. Hence every $\bar{R}(d)$-regular element is $\bar{R}$-regular and $Q(\bar{R}(d)) \subset Q(\bar{R})$. Similarly $Q(R(d))$ $\subseteq \mathrm{Q}(\mathrm{R})$.

Let $q_{i}(d)=q_{i} \cap \bar{R}(d)$ for $i=1, \cdots, l$. Then $q_{1}(d) \cap \cdots \cap q_{\ell}(d)=0$. We claim that there are no containments amongst these primes. For suppose $q_{i}(d)$ $\subset q_{j}(d)$. Let $\alpha$ be a homogeneous element of $q_{i}$. Then $\alpha^{d} \varepsilon q_{i}(d) \subseteq q_{j}(d)$ implies $\alpha \varepsilon q_{j}$. Since $q_{i}$ is generated by homogeneous elements $q_{i} \subseteq q_{j}$ and we must have $i=j$. Hence $q_{1}(d), \cdots, q_{\ell}(d)$ are the minimal primes of $\bar{R}(d)$. Similarly, $\rho_{i}(d), \cdots, \rho_{\ell}(d)$ are the minimal primes of $R(d)$.

We wish to see that $Q(R(d))=Q(\bar{R}(d))$ and that $\bar{R}(d)$ is the integral closure of $R(D)$ in its total quotient ring. Now $Q(R(d))=Q\left(R(d) / \rho_{1}(d)\right) \times \ldots \times\left(R(d) / \rho_{\ell}(d)\right)$ $=Q\left(R / \rho_{1}(d)\right) \times \cdots \times Q\left(R / \rho_{\ell}(d)\right)$ and $Q(\bar{R}(d))=Q\left(\bar{R}(d) / q_{1}(d)\right) \times \cdots \times Q\left(\bar{R}(d) / q_{\ell}(d)\right)=$ $Q\left(\bar{R} / q_{1}(d)\right) \times \cdots \times\left(\bar{R} / q_{\ell}(d)\right)$. So it suffices to establish the result when $R$ is an integral domain and $R_{0} \neq R$.

Let $K=Q(R)$ and let $T=R^{h}-\{0\}$. Let $J=\left\{n \varepsilon \mathbb{Z} \mid\left(T^{-1} \dot{R}\right)_{n} \neq 0\right\}$. Then $J$ is an additive subgroup of $\mathbb{Z}$ and hence $J=m \mathbb{Z}$ for some positive integer $m$. Let $e=1 \mathrm{~cm}(d, m)$ and set $f=e / m$. Then $R(d)=R(e)=R(f m)=R(m)(f)$ and $\bar{R}(d)$ 
$=\bar{R}(e)=\bar{R}(f m)=\bar{R}(m)(f)$. Thus replacing $R$ and $\bar{R}$ by $R(m)$ and $\bar{R}(m)$, respectively, we may assume there exists a nonzero degree 1 homogeneous element $t$ in $\mathrm{T}^{-1} \mathrm{R}$.

Let $L=\left(T^{-1} R\right)_{0}$. Then we may identify $R$ with a graded subring of $L\left[t, t^{-1}\right]$ where $a$; $R_{n}$ is identified with $\left(a t^{-n}\right) t^{n}$. Since $t$ has degree 1 , $t$ is transcendental over $L$ and $L[t]$ is a polynomial ring over $L$. Since $L\left[t, t^{-1}\right]$ is the localization of $\mathrm{L}[\mathrm{t}]$ with respect to powers of $t$ it is integrally closed in $K$ and hence $\bar{R}$ is contained in $L\left[t, t^{-1}\right]$ as a graded subring.

Thus $R(d) \subseteq \bar{R}(d) \subseteq L\left[t^{d}, t^{-d}\right]$. We claim that $L \subseteq Q(R(d))$. For if $a, b \varepsilon R R_{n}$ and $b \neq 0$ then $a b^{d-1}, b^{d} \varepsilon R(d)_{n}$ and $a / b=a b^{d-1} / b^{d}$. Hence $L\left[t^{d}, t^{-d}\right] \subseteq Q(R(d))$ and $Q(R(d))=Q(\vec{R}(d))=L\left(t^{d}\right)$.

Clearly $\bar{R}(d)$ is integral over $R(d)$. Let $\beta \varepsilon \bar{R}(d)_{n}$ so that $B$ is integral over $R$. Say $\beta^{m}+a_{1} \beta^{m-1}+\cdots+a_{m-1} \beta+a_{m}=0$ where $a_{i} \varepsilon R^{n} \quad(i=1, \cdots, m)$. For each $i$ let $c_{i}$ denote the homogeneous component of $a_{i}$ of degree ind. Then $\beta^{\mathrm{m}}+\mathrm{c}_{1} \beta^{\mathrm{m}-1}+\cdots+\mathrm{c}_{\mathrm{m}-1} \beta+\mathrm{c}_{\mathrm{m}}=0$ and $c_{1}, \cdots, \mathrm{c}_{\mathrm{m}} \varepsilon \mathrm{R}(\mathrm{d})$ so that $\beta$ is integral over $R(d)$. Thus to finish the proof it suffices to see that $\bar{R}(d)$ is integrally closed in $L\left[t^{d}, t^{-d}\right]$.

If $\gamma^{\circ} L\left[t^{d}, t^{-d}\right]$ is integral over $\bar{R}(d)$ then $\gamma$ is integral over $R(d)$ and hence over $R$. Thus $\gamma=\bar{R} \cap L\left[t^{d}, t^{-d}\right]=\bar{R}(d)$ since $\bar{R}$ is a graded subring of $L\left[t, t^{-1}\right]$.

We will now look at the question of seminormality for a reduced graded ring $R$ as above. We first recall the relevant definitions.

For a ring $A$ let $J(A)$ denote the Jacobson radical of $A$ and let $\bar{A}$ denote the normalization of $A$. The seminormalization ${ }^{+} A$ of $A$ is defined by

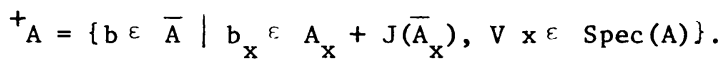

The ring $A$ is said to be seminormal if $A={ }^{+} A$. There is also a relative notion. For an integral extension of rings $A \subseteq B$, the seminormalization ${ }_{B}^{+}$is defined by

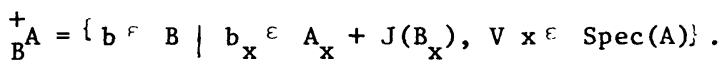

We say that $A$ is seminormal in $B$ if $A={ }_{B}^{+} A$. We refer the reader to [5] for some of the fundamental results on seminormality.

Recall that Hamann's criterion for seminormality asserts that $A$ is seminormal in $B$ if and only if $A$ contains each element $b$ of $B$ such that $b^{2}, b^{3} \varepsilon A$ ([1], Prop.1.4). In ([4], Thm. 2) Anderson adapts an argument of Brewer, Costa and McCrimmon ([5], Thm.1) to show that a graded integral domain $R=R_{0}^{\oplus} R_{1}^{A} \ldots$ is seminormal if and only if $R$ contains each homogeneous element $s \in \bar{R}$ such that $s^{2}, s^{3} \varepsilon R$. We note that the same argument applies to any integral extension of rings $\mathrm{R} \subseteq \mathrm{S}$ such that $\mathrm{S}$ is a graded ring and $\mathrm{R}$ is a graded subring of $\mathrm{S}$ (we say that $\mathrm{R} \subseteq \mathrm{S}$ is an integral extension of graded rings).

PROPOSITION 2.4. Let $R \subset S$ be an integral extension of graded rings. Then $R$ is seminormal in $S$ if and only if $R$ contains each homogeneous element $s$ of $S$ such that $s^{2}, s^{3} \varepsilon R$.

PROOF. One half of the assertion follows immediately from Hamann's criterion. Thus it suffices to show that if $R$ contains each homogeneous element of $S$ whose square and cube is in $R$ then $R$ is seminormal in $S$. 
Assume that this condition is valid but $R$ is not seminormal in $S$. Thus there exists an element $s$ of $s \backslash R$ such that $s^{2}, s^{3} \varepsilon$. For an element $s$ of $s$ let $\ell(s)$ denote the number of nonzero homogeneous components of $s$. For each nonzero element $s=s_{i(1)}+\cdots+s_{i(m)}$ of $s$ such that $0 \neq s_{i(j)} \in s_{i(j)}$ and $i(1)<\cdots<i(m)$ let $p(s)=\max \left\{k \mid s_{i(1)}, \cdots, s_{i(k-1)} \varepsilon R\right\}$. In particular if $p(s)=1$ then the initial nonzero homogeneous component of $s$ is not in $R$.

Amongst those elements of $S \backslash R$ whose square and cube are in $R$ choose an element $s$ with $\ell(s)$ minimal and such that if $t$ has the same properties then $p(t) \leq p(s)$.

Write $s=s_{i(1)}+\cdots+s_{i(m)}$ as above so that $m=\ell(s)$. Since $s^{2}=s_{i(1)}^{2}+$ (higher degree homogeneous terms) is in $R$ we must have $s_{i(1)}^{2} R$. Similarly $\mathrm{s}_{\mathrm{i}(1)}^{3} \varepsilon \mathrm{R}$ and hence $\mathrm{s}_{\mathrm{i}(1)} \varepsilon \mathrm{R}$. Thus $\mathrm{k}=\mathrm{p}(\mathrm{s}) \geq 2$.

Now $\left(2 s_{i(1)} s\right)^{2}$ and $\left(2 s_{i(1)} s\right)^{3}$ are in $R$ and $\ell\left(2 s_{i(1)} s\right) \leq(s)$. Since the $(i(1)+i(k))$-component of $s^{2}$ is $2 s_{i(1)} s_{i(k)}+$ (terms in $R$ ) we must have $2 s_{i(1)} s_{i(k)} \& R$. If $2 s_{i(1)} s_{i(j)}=0$ for some $j$ then $\ell\left(2 s_{i(1)} s\right)<\ell(s)$ and hence $2 s_{i(1)} s$ R. If $2 s_{i(1)} s_{i(j)} \neq 0$ for all $j$ then $\ell\left(2 s_{i(1)} s\right)=\ell(s)$ and $?\left(2 s_{i(1)} s\right)>p(s)$ so that $2 s_{i(1)} s$. Thus in either case $2 s_{i(1)} s \quad R$. Now $\left(3 s_{i(1)}^{2} s\right)^{2}$ and $\left(3 s_{i(1)}^{2} s\right)^{3}$ are in $R$ and $\chi\left(3 s_{i(1)}^{2} s\right) \leq \mathcal{L}(s)$. Since the $(2 i(1)+i(k))$-component of $s^{3}$ is $3 s_{i(1)}^{2} s_{i(k)}+$ (terms in $R$ ) we must have $3 s_{i(1)}^{2} s_{i(k)} \quad R$. If $3 s_{i(1)}^{2} s_{i(j)}=0$ for some $j$ then $\ell\left(3 s_{i(1)}^{2} s\right)<\chi(s)$ and hence $3 s_{i(1)}^{2}$ R. If $3 s_{i(1)}^{2} s_{i(j)} \neq 0$ for all $j$ then $\ell\left(3 s_{i(1)}^{2} s\right)=\chi(s)$ and $p\left(3 s_{i(1)}^{2} s\right)>p(s)$ so that $3 s_{i(1)}^{2} s$. Again in either case $3 s_{i(1)}^{2} s$.

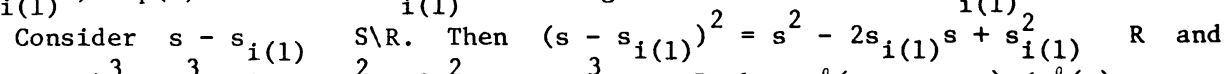
$\left(s-s_{i(1)}\right)^{3}=s^{3}-3 s_{i(1)} s^{2}+3 s_{i(1)}^{2} s-s_{i(1)}^{3}{ }^{\prime}$ but $\ell\left(s-s_{i(1)}\right)<\ell(s)$, a contradiction.

Hence $R$ is seminormal in $S$.

COROLLARY 2.5. Let $R$ be a reduced graded ring with a finite number of minimal primes. Then the seminormalization ${ }^{+} R$ of $R$ is a graded ring and contains $R$ as a graded subring.

PROOF. By 2.1 the normalization $\bar{R}$ of $R$ is graded and contains $R$ as a graded subring. Let $R^{\prime}=\sum_{n \varepsilon \bar{y}}{ }^{+} R \cap \bar{R}_{n}$. By $2.4 R^{\prime}$ is seminormal. But ${ }_{R}$ is the smallest seminormal subring of $\bar{R}$ that contains $R$ and hence $R^{\prime}={ }^{+} R$ is a graded subring of $\bar{R}$. Since $R$ is a graded subring of $\bar{R}$ we know that $R$ is graded subring of ${ }^{+} R$.

In order to prove the analogue of 2.3 for the seminormalization of $R$ we adapt a construction of Swan ([2], Thm. 4.1) to describe ${ }_{R}^{+}$.

Let $R \subseteq S$ be an integral extension of graded rings (so that $R$ is a graded subring of $S$ ) and let $T$ be a graded subring of $S$ containing $R$. Let $\left\{s_{\alpha} \mid \alpha \varepsilon A\right\}$ be a well ordering of the set of all homogeneous elements of $S-T$ whose square and whose cube are in $T$. We inductively define graded subrings $T_{\alpha}$ of $S$ such that $\mathrm{T}_{\alpha} \subseteq \mathrm{T}_{\beta}$ whenever $\alpha \leq \beta$. Let $\mathrm{T}_{-1}=\mathrm{T}, \mathrm{T}_{\alpha+1}=\mathrm{T}_{\alpha}\left[\mathrm{s}_{\alpha}\right]$ and for a limit ordinal $\beta$ let $T_{\beta}=\cup_{\alpha<\beta} T_{\alpha}$. Set $T^{\prime}=\cup_{\alpha \varepsilon \Lambda_{\alpha}} T_{\alpha}$ so that $T^{\prime}$, is again a graded subring of $S$ containing $R$. Now let $R^{(0)}=R, R^{(n+1)}=R(n)^{\prime}$ and set $R^{\star}=\cup_{n \geq 0} R^{(n)}$. Note that $R^{*}$ is also a graded subring of $S$ containing $R$. 
PROPOSITION 2.6. Let $R \subseteq S$ be an integral extension of graded rings. With notation as above ${ }_{S}^{+} R=\bigcup_{n \geq 0}{ }^{(n)}$. In particular $R$ is a graded subring of $S$. PROOF. As observed earlier $R^{*}=U_{n \geq 0} R^{(n)}$ is a graded subring of $S$ and the seminormality of $R^{*}$ in $S$ follows from 2.4. Thus it suffices to see that $R^{*} \subset \mathrm{S}^{\mathrm{R}}$. Now $R=R{ }^{(0)} \subseteq{ }_{S}^{+} R$. Suppose $T=R{ }^{(n)} \subseteq{ }_{S}^{+} R$. Let $\left\{s_{\alpha} \mid \alpha \varepsilon \varepsilon\right\}$ be a well ordering of the set of all homogeneous elements of $\mathrm{S}-\mathrm{T}$ whose square and whose cube are in $T$. Define $T_{\alpha}$ as above. We show by transfinite induction that $\mathrm{T}_{\alpha} \subseteq{ }_{\mathrm{S}}^{+}$for all $\alpha$. Now ${ }^{\mathrm{T}}{ }_{-1}=\mathrm{T} \subseteq{ }_{\mathrm{S}}^{+} \mathrm{R}$ by assumption. Suppose $\beta>-1$ and that $\mathrm{T}_{\alpha} \subseteq \stackrel{\mathrm{S}}{\mathrm{R}}_{\mathrm{R}}$ for all $\alpha<\beta$. If $\beta=\alpha+1$ then $\mathrm{T}_{B}=\mathrm{T}_{\alpha}\left[\mathrm{s}_{\alpha}\right]$ where $\mathrm{s}_{\alpha}^{2}, \mathrm{~s}_{\alpha}^{3} \varepsilon \mathrm{T}$. Since

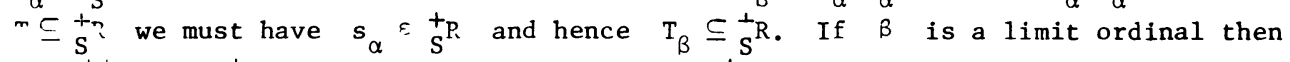

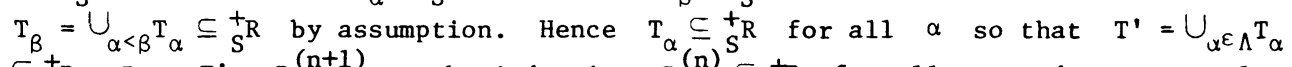
$\subseteq^{B}{ }_{S}$. But $T^{\prime}=R^{(n+1)}$ so by induction $R^{(n)} \subseteq{ }_{S}^{R}$ for all $n$ and consequently $R^{*} \subseteq{ }_{S}^{+} R$. Since ${ }_{S}^{+}$is the smallest subring of $S$ that contains $R$ and is seminorma 1 in $S$ we must have $R^{*}={ }_{S}^{+} R$.

PROPOSITION 2.7. Let $R$ be a reduced graded ring having only a finite number of minimal primes. For each positive integer $d \quad\left({ }^{+} R\right)(d)$ is the seminormalization of $R(d)$.

PROOF. Let $\bar{R}$ denote the normalization of $R$ and let $d$ be a positive integer. Then $\vec{R}(d)$ is the normalization of $R(d)$ by 2.3. Since we have inclusions of graded rings $R \subseteq{ }^{+} \subseteq S$ we also have $R(d) \subseteq\left({ }^{+} R\right)(d) \subseteq \bar{R}(d)$ and $\left(^{+} R\right)(d)$ is seminormal by 2.4. Thus it suffices to see that $\left({ }^{+} R\right)(d) \subseteq{ }^{+}(R(d))$. Using the notation of 2.6 we have ${ }^{+} R=\bigcup_{n>0} R^{(n)}$. Thus it suffices to see that
$R^{(n)}$ (d) $\subseteq^{+}(R(d))$ for all $n$. Now $R^{(0)}(d) \subseteq R(d) \subseteq{ }^{+}(R(d))$. Let $n^{>} 0$ and assume that $R^{(n)}(d) \subseteq{ }^{+}(R(d))$. Let $T=R^{(n)}$ and let $\left\{s_{\alpha} \mid \alpha \varepsilon \Lambda\right\}$ be a well ordering of the set of all homogeneous elements of $\bar{R}-\mathrm{T}$ whose square and whose cube are in $T$. Define $T_{\alpha}$ as above. We need to show that $T_{\alpha}(d) \subseteq{ }^{+}(R(d))$ for all $\alpha$.

Since $T_{-1}-T$ we have $T_{-1}(d) \subseteq{ }^{+}(R(d))$. Let $\beta>-1$ and suppose that $\mathrm{T}_{\alpha}(\mathrm{d}) \subseteq^{+}(\mathrm{R}(\mathrm{d}))$ for all $\alpha<\beta$. If $\beta=\alpha+1$ then $\mathrm{T}_{\beta}=\mathrm{T}_{\alpha}\left[\mathrm{s}_{\alpha}\right]$. Let $\mathrm{s}_{\mathrm{k}}=\mathrm{s}_{\alpha}$ and suppose $t \in \mathrm{T}_{\beta}(\mathrm{d})$ is homogeneous of degree $\ell$. Then $t=\Sigma_{\mathbf{r}_{\mathbf{k}}} \mathrm{s}^{\mathrm{k}}$ where $\mathbf{r}_{k} \varepsilon \mathrm{T}_{\alpha}^{\mathrm{h}}$ and $\operatorname{deg}\left(r_{k}\right)=\ell_{d}-k \cdot \operatorname{deg}(s)$ for all $k$. Now $\left(r_{k} s^{k}\right)^{2},\left(r_{k} s^{k}\right)^{3} \varepsilon T_{\alpha}(d) \subseteq{ }^{+}(R(d))$ and $r_{k} s^{k} \bar{R}(d)=\overline{R(d)}$ (by 2.3) implies that $r_{k} s_{\varepsilon}+(R(d))$ for each $k$ and hence $t \varepsilon{ }^{+}(R(d))$. Since $t$ was an arbitrary homogeneous element of $T_{B}(d)$ we have $T_{B}(d)$ $\subseteq{ }^{+}(R(d))$. If $\beta$ is a limit ordinal then $T_{\beta}=\bigcup_{\alpha<\beta} T_{\alpha}$ so that $T_{\beta}(d) \subseteq{ }^{+}(R(d))$ by assumption. Hence $\mathrm{T}_{\alpha}(\mathrm{d}) \subseteq^{+}(\mathrm{R}(\mathrm{d}))$ for all $\alpha$ and since $\mathrm{R}^{(\mathrm{n}+1)}=\bigcup_{\alpha} \Lambda^{\mathrm{T}} \alpha$ we also have $R^{(n+1)}(d) \subseteq{ }^{+}(R(d))$.

Thus $R^{(n)}(d) \subseteq^{+}(r(d))$ for all $n$ and $\left({ }^{+} R\right)(d)=\cup_{n^{\prime} 0^{R}}{ }^{(n)}(d) \subseteq{ }^{+}(R(d))$ as desired. Consequently ${ }^{+}(\mathrm{R})(\mathrm{d})={ }^{+}(\mathrm{r}(\mathrm{d}))$.

LEMMA 2.8. Let $R$ be a reduced graded ring having only a finite number of minimal primes and suppose that $t \in R_{1}$ is invertible. Then $(\bar{R})_{0}$ is the normalization and $\left({ }^{+}\right)_{0}$ is the seminormalization of $R_{0}$.

PROOF. To simplify notation let $A=R_{0}, B=\left({ }^{+} R\right)_{0}$ and $C+(\bar{R})_{0}$. Define a $C$-algebra map $\varphi: C\left[X, X^{-1}\right] \rightarrow \bar{R}$ by $\varphi(X)=t$. Then $c p$ is a degree preserving isomorphism of graded ring such that $\varphi\left(B\left[X, X^{-1}\right]\right)={ }^{+} R$ and $p\left(A\left[X, X^{-1}\right]\right)=R$. 
Thus $C\left[X, X^{-1}\right]$ is normal and hence $C$ is normal. Let $T$ denote the set of homogeneous R-regular elements of $R$. By 2.2 we know that $\bar{R}$ is a graded subring of $T^{-1} R$. So an element of $C$ is of the form $\frac{r}{s}$ where $r$ and $s$ are homogeneous elements of $R$ of the same degree, $s$ is $R$-regular and $\frac{r}{s}$ is integral over $R$. Let $n=\operatorname{deg}(r)$. Then $r t^{-n}$, $s t^{-n} \varepsilon R_{0}=A$ and $\frac{r}{s}=\frac{r t^{-n}}{s t^{-n}} \varepsilon Q(A)$. A direct computation shows that $\frac{r}{s}$ is integral over $A$ and hence $A \subseteq C \subseteq \bar{A}$. Since $C$ is normal we must have $\mathrm{C}=\overline{\mathrm{A}}$.

Since $\mathrm{B}\left[\mathrm{X}, \mathrm{X}^{-1}\right] \simeq{ }^{+} \mathrm{R}$ is seminormal if $\alpha \varepsilon \mathrm{Q}(\mathrm{B})$ and $\alpha^{2}, \alpha^{3} \varepsilon \mathrm{B}$ then $\alpha E{ }_{R} R \cap(B)=B$. Thus $B$ is seminormal by Hamann's criterion. Thus we need only show that $B \subseteq{ }^{+} A$. We already have $B \subseteq C=\bar{A} \subseteq Q(A)$ and $B\left[X, X^{-1}\right]$ is the seminormalization of $A\left[X, X^{-1}\right]$. If $P_{1}$ and $P_{2}$ are prime ideals in $B$ lying over the same prime ideal of $A$ then $P_{1} B\left[X, X^{-1}\right]=P_{2} B\left[X, X^{-1}\right]$ so that $P_{1}=P_{2}$. Let $P$ be a prime ideal of $B$ and let $\rho=P \cap A$. Then the canonical map $\kappa(\rho) \rightarrow \kappa(P)$ must be an isomorphism since $K(\rho)(X) \rightarrow K(P)(X)$ is an isomorphism. Hence $B \subseteq{ }^{+} A$ by Traverso's characterization of ${ }^{+} \mathrm{A}$ ([ ], Prop. 1.3).

We can show that normalization (respectively, seminormalization) and homogeneous localization commute.

COROLLARY 2.9. Let $R$ be a reduced graded ring having only a finite number of minimal primes and let $f \neq 0$ be homogeneous of positive degree $d$. Then $\bar{R}_{f}$ is the normalization and $\left({ }^{+} R\right)_{(f)}$ is the seminormalization of $R_{(f)}$.

PROOF. $R_{(f)}$ is the degree 0 subring of $R_{f}(d)$ and $f \in R_{f}(d)$ is invertible and is homogeneous of degree $1 . \bar{R}_{f}(d)$ is the normalization of $R_{f}$ (d) by 2.3 and $\left({ }^{+} R\right)_{f}(d)$ is the seminormalization of $R_{f}(d)$ by 2.7 . So the assertion follows immediately from applying 2.8 to the reduced graded ring $R_{f}(d)$. (Note that $R_{f}$ (d) has only a finite number of minimal primes by the proof of 2.3.)

REMARKS. D. F. Anderson [6] shows that if $A$ is a seminormal integral domain and $I$ an invertible fractional ideal then the Rees algebra $R(I)=\oplus I^{n}$ is again seminormal. $n \geq 0$

Let $A$ be a reduced ring with a finite number of minimal primes and assume that $A$ is seminormal. Let $I$ be an ideal of $A$. It is natural to ask what conditions on I guarantee us that the Rees algebra $R(I)$ (or the scheme Proj(R(I)) is again seminormal. We do not have any satisfactory answer to these questions at this time but we would like to make some observations.

(2.10) Since $\mathrm{A}$ is a reduced ring with finitely minimal primes the seminorma1ity of $A$ implies the seminormality of the polynomial ring $A[t]$ ([5], Thm. 2 or use 2.2 and 2.4). Now $R(I) \simeq A[I t]$ is a graded subring of $A[t]$ and has the same total quotient ring as $A[t]$. Thus $R(I)$ is seminormal if and only if $A[I t]$ is seminormal in $\mathrm{A}[\mathrm{t}]$.

(2.11) By 2.4 we see that $A[I t]$ is seminormal in $A[t]$ if and only if for each positive integer $n$ and each element $a \varepsilon A$ if $a^{2} \varepsilon I^{2 n}$ and $a^{3} \varepsilon I^{3 n}$ then a $\varepsilon I^{n}$. (cf. [6] in case $A$ is an integral domain.)

(2.12) Say an ideal $J$ of a ring $A$ is pseudo-reduced if whenever a $\varepsilon$ and $a^{2}, a^{3} \varepsilon J$ then $a \varepsilon J$. If $A$ and $I$ are as above when are all powers of I pseudo-reduced? 
If $A$ is a seminormal domain and $I$ is an invertible ideal then so is each power of $I$. Hence $A:\left(A: I^{n}\right)=A$ and $I^{n}$ is pseudo-reduced for all $n \geq 1$ (this is Anderson's argument in [6]).

$$
\text { If } \mathrm{gr}_{\mathrm{I}}(\mathrm{A})={ }_{\mathrm{n}}^{>} \mathrm{I}^{\mathrm{n}} / \mathrm{I}^{\mathrm{n}+1} \text { is reduced then all powers of } \mathrm{I} \text { are pseudo-reduced. }
$$

So, for example, if $I$ is a reduced ideal of $A$ that is generated by an A-regular sequence then the Rees algebra $R(I)$ is again seminormal. We would like to point out that an analagous result for normality was proven by Barshay [7]. Namely, if A is a normal domain and $\mathrm{gr}_{\mathrm{I}}(\mathrm{A})$ is reduced then $\mathrm{R}(\mathrm{I})$ is again a normal domain.

\section{IMPLICATIONS FOR PROJECTIVE VARIETIES.}

Let $k$ be a fixed algebraically closed field of characteristic 0 . When we use the term variety we assume that the underlying topological space is the set of closed points of a reduced, separated scheme of finite type over $k$.

Let $U$ be an open subset of a variety $\left(X, \theta_{X}\right)$. A $k$-valued function on $U$ is said to be c-regular if it is continuous and regular on the nonsingular points of $U$. Let $O_{X}^{c}$ denote the sheaf of c-regular functions on $X$. We say that $X$ is weakly normal at $x \in X$ if $\theta_{x, x}=\theta_{x, x}^{c}$ and that $x$ is weakly normal if $\theta_{x}=\theta_{x}^{c}$. An affine variety is weakly normal if and only if its affine coordinate ring is seminormal ([1], Thm. 2.2 and (2.7)). For the fundamental results concerning weakly normal varieties the reader can consult [1].

Let $\mathrm{X} \subset \mathrm{P}^{\mathrm{n}}$ be a projective variety and let $\mathrm{R}=\mathrm{k}\left[\mathrm{x}_{0}, \cdots, \mathrm{x}_{\mathrm{n}}\right]$ denote the homogeneous coordinate ring of $X$ with respect to the given embedding. Then no minimal prime of $R$ contains the irrelevant maximal ideal $\left(x_{0}, \cdots, x_{n}\right)$. Thus letting $T$ denote the set of homogeneous R-regular elements in $R$ we know that $\bar{R}$ is a graded subring of $T^{-1} R$ by 2.2. Hence ${ }^{+}{ }_{R}$ is a graded subring of $T^{-1} R$ by 2.6.

Now if $X$ has $l$ irreducible components then $\bar{R}_{0} \simeq \Pi_{i=1}^{l}$. This is not the case with ${ }_{\mathrm{R}}$.

LEMMA 3.1. Let $R$ be the homogeneous coordinate ring of a projective variety $X \subset \mathbb{P}^{n}$ and suppose that $R$ is not seminormal. Then there exist positive degree homogeneous elements $s_{1}, \cdots, s_{m}$ in $\bar{R}$ such that $s_{i}^{2}, s_{i}^{3} \varepsilon R\left[s_{1}, \cdots, s_{i-1}\right]$ but $s_{i}$ $\notin \mathrm{R}\left[\mathrm{s}_{1}, \cdots, \mathrm{s}_{i-1}\right]$ for $i=1, \cdots, \mathrm{m}$ and $t_{\mathrm{R}}=\mathrm{R}\left[\mathrm{s}_{1}, \cdots, \mathrm{s}_{\mathrm{m}}\right]$. In particular $\left({ }_{\mathrm{R}}^{+}\right)_{0}=k$.

PROOF. Since $R$ is not seminormal by 2.4 there exists a homogeneous element $s_{1} \varepsilon \bar{R}-R$ whose square and whose cube is in $R$. Since $k=R_{0} \subset \bar{R}_{0} \simeq \Pi_{i=1}^{l} k$ (where $\ell$ is the number of minimal primes of $\bar{R}$ ) is the diagonal mapping we must have $\operatorname{deg}\left(s_{1}\right)>0$. Let $R_{1}=R\left[s_{1}\right]$. Then $R_{1}$ is a graded subring of $\bar{R}$ (and of $t_{R}$ ) whose degree 0 homogeneous piece is $k$. If $R_{1}$ is not seminormal there exists a homogeneous element $s_{2} \varepsilon \vec{R}-R_{1}$ whose square and whose cube is in $R_{1}$. As above we must have $\operatorname{deg}\left(s_{2}\right)>0$ so that if $R_{2}=R_{1}\left[s_{2}\right]$ then $R_{2}$ is a graded subring of $\bar{R}$ whose degree 0 homogeneous piece is $k$.

Continuing in this fashion we obtain an increasing chain of graded subrings of $\bar{R}: R<R_{1}<R_{2}<\cdots$ each of whose degree 0 homogeneous piece is $k$. Since $\bar{R}$ is a finite R-module ([3], vo1. 1, Thm. 9, p. 267) this process must end in a finite number of steps. If $R_{m}$ is the last ring in the chain then $R_{m}$ is seminormal by 2.4. Clearly $R_{m} \subset{ }^{+}{ }_{R}$ and since ${ }_{R}$ is the smallest seminormal subring of $\bar{R}$ 
that contains $R$ we must have $R_{m}={ }^{+}$.

It is well known that the normalization of a projective variety is again projective. Most proofs assume that the original variety is irreducible (e.g. [8] Them. 4, p. 400). The general case follows quickly from the irreducible case. We first take the normalization of each irreducible component and then construct the disjoint union of these normal projective varieties observing that this union can be embedded in some projective space. We would like to point out that if $\mathrm{X} \subset \mathrm{P}^{\mathrm{n}}$ has $\ell$ irreducible components and $\ell>1$ then the homogeneous coordinate ring of the normalization of $\mathrm{X}$ cannot be realized as the normalization of some Veronese subring of the homogeneous coordinate ring $R=k\left[x_{0}, \cdots, x_{n}\right]$ of $x$. For the normalization of $R$ (and of every Veronese subring of $R$ ! has as its degree 0 humogeneous piece $\prod_{i=1}^{l} k$ whereas the homogeneous coordinate ring of any projective variety has $k$ as its degree 0 homogeneous piece.

We now show that the weak normalization of a projective variety is again projective. We include a complete proof for the convenience of the reader.

PROPOSITION 3.2. The weak normalization of a projective variety is a projective variety.

PROOF. Let $x=\mathbb{P}^{n}$ be a projective variety with homogeneous coordinate ring $R=k\left[x_{0}, \cdots, x_{n}\right] / I=k\left[x_{0}, \cdots, x_{n}\right]$. Let $S$ denote the seminormalization of $R$. Then $S$ is a graded ring (2.5) and $S_{0}=k$ by 3.1. Hence there exists a positive integer d such that $\mathrm{S}(\mathrm{d})$ is generated, as a k-algebra, by degree 1 homogeneous elements ([8], Lemma, p. 403). Now $S(d)$ is the seminormalization of $R(d)$ by 2.7 and $R(d)$ is the homogeneous coordinate ring of the Segre transform $\Phi_{d}(X)$ of $X$. Replacing $\mathrm{X}$ by $\Phi_{\mathrm{d}}(\mathrm{X})$ we may assume that $\mathrm{S}$ is generated as a k-algebra by degree 1 homogeneous elements.

Let $\mathrm{y}_{0}, \cdots, \mathrm{y}_{\mathrm{N}}$ be a $\mathrm{k}$-basis for $\mathrm{s}_{1}$ and let $\mathrm{Y}_{\mathrm{O}}, \cdots, \mathrm{Y}_{\mathrm{N}}$ be indeterminates. Define a degree preserving map of graded $k$-algebras $P: k\left[Y_{0}, \cdots, Y_{N}\right] \rightarrow S$ by $i\left(Y_{i}\right)$ $-\mathrm{y}_{i}$ for $\mathbf{i}=0, \cdots, N$ and let $\mathrm{J}=$ ker $\rho$. Let $\mathrm{Y}-\mathbb{P}^{N}$ be the projective variety defined by $J$. Since $R$ is a graded subring of $S$ the elements $x_{0}, \cdots, x_{n}$ are in $\mathrm{S}_{1}$. Let $\mathrm{F}_{0}, \cdots, \mathrm{F}_{\mathrm{n}}$ be linear forms whose images in $\mathrm{s}$ are $\mathrm{x}_{0}, \cdots, \mathrm{x}_{\mathrm{n}}$, respectively, and let $L \subseteq \mathbb{P}^{N}$ be the linear subspace defined by the ideal $\left(F_{0}, \cdots, F_{n}\right)$. Let $p: \mathbb{P}^{N}-L \sim \mathbb{P}^{n}$ be the projection defined by $p(a)=\left(F_{0}(a), \cdots, F_{n}(a)\right)$. Since $R \subset s$ is an integral extension of graded rings $Y \cap L=p$ (see [8], Proof of Thm. 4.1, p. 405) and $\pi=\left.\mathrm{P}\right|_{\mathrm{Y}}: \mathrm{Y} \rightarrow \mathbb{P}^{\mathrm{n}}$ is a finite morphism ([8], Prop. 6, p. 246). Then $\pi(\mathrm{Y})$ is the projective variety defined by the homogeneous ideal $\mathrm{J} \cap \mathrm{k}\left[\mathrm{X}_{\mathrm{o}}, \cdots, \mathrm{X}_{\mathrm{n}}\right]$ = I, i.e., $\pi(Y)=X$.

Let $f=R_{1}$ be an R-regular element. Then $Q\left(R_{(f)}\right)=Q\left(S_{(f)}\right)$ are the function fields of $X$ and $Y$, respectively, so that $\pi$ is birational. Let $U_{i}=X \| \mathbb{P}_{X_{i}}^{n}$ and $v_{i}=\pi^{-1}\left(U_{i}\right)$ for $i=0, \cdots, n$. Then $U_{i}$ and $v_{i}$ are affine with affine coordinate rings $R_{\left(x_{i}\right)}$ and $S_{\left(x_{i}\right)}$, respectively, and $S_{\left(x_{i}\right)}$ is the seminormalization of $R_{\left(x_{i}\right)}$ by 2.9. Thus $\left(v_{i}, \pi \mid v_{i}\right)$ is the weak normalization of $U_{i}$ for $i=0, \cdots, n$ and hence $Y=U_{i=0}^{n} V_{i}$ is weakly normal and $(Y, \pi)$ is the weak normalization of $\mathrm{X}$.

DEFINITION 3.3. We say that a projective variety $X \subset \mathbb{P}^{n}$ is arithmetically weakly normal if its homogeneous coordinate ring with respect to this embedding is 
seminormal.

REMARK 3.4. Suppose $\mathrm{X}=\mathbb{P}^{\mathrm{n}}$ is arithmetically weakly normal and let $R$ denote its homogeneous coordinate ring with respect to this embedding. Then $R_{f}$ is seminormal for each $0 \neq f \in R_{1}$ by 2.8. Since $R_{(f)}$ is the affine coordinate ring of the affine open subset $x_{f}$ of $X$ and these cover $X$ (as $f$ ranges through nonzero elements of $R_{1}$ ) we see that $X$ is weakly normal.

PROPOSITION 3.5. Let $\mathrm{X} \subset \mathbf{P}^{\mathrm{n}}$ be a projective variety. Then $\mathrm{X}$ is weakly normal if and only if its Segre transforms ${ }^{d}(X)$ are arithmetically weakly normal for $d$ sufficiently large.

PROOF. Let $R$ denote the homogeneous coordinate ring of $X-\mathbb{P}^{n}$. Suppose that $X$ is weakly normal. Then $X_{f}$ is weakly normal for each $0 \neq f \varepsilon R_{1}$ so that $R_{(f)}$ is seminormal for each $0 \neq f \in R_{1}$. Thus $R_{f}$ is seminormal for each $0 \neq \mathrm{f}_{\varepsilon} \mathrm{R}_{1}$ by 2.8. Hence $\mathrm{R}_{\mathrm{f}}=\left({ }^{+} \mathrm{R}\right)_{\mathrm{f}}$ for each $0 \neq \mathrm{f} \varepsilon \mathrm{R}_{1}$ and letting $\mathrm{m}=\mathrm{R}_{+}$ we know that $\operatorname{Supp}\left({ }^{+} R / R\right) \sim\{\mathrm{m}\}$. Since ${ }_{R / R}$ is a finitely generated graded R-module this implies that $\left({ }^{+} R / R\right)_{m}=0$ for $m$ sufficiently large, i.e., $R_{m}={ }_{R_{m}}$ for $m$ sufficiently large. Now $R_{0}={ }^{+} R_{0}=k$ by 3.1 so that $R(d)=\left({ }^{+} R\right)(d)$ for $d$ sufficiently large and the latter is seminormal by 2.2 and 2.4 . Since $R(d)$ is the homogeneous coordinate ring of the Segre transform $\Phi_{d}(X)$, the Segre transforms ${ }^{2} \mathrm{~d}(\mathrm{X})$ are weakly normal for $d$ sufficiently large.

The converse is clear by 3.4 as $\Phi_{d}(X)$ is isomorphic to $X$.

REMARK 3.6. We again remind the reader that the analogous statement for a reducible normal variety is false. For if $X \subset \mathbb{P}^{n}$ is a reducible normal projective variable with $x$ irreducible components and $R$ is its homogeneous coordinate ring then $R(d)_{0}=R_{0}=k$ whereas $\bar{R}(d)_{0}=\bar{R}_{0}=\mu_{i=1}^{x} k$ for each $d>0$. Since $\bar{R}(d)$ is the normalization of $R(d)$ by 2.2 we know that $R(d)$ is not normal for all $d>0$. Geometrically this is easy to see because $R(d)$ is the affine coordinate ring of the union of $x$ cones through the origin in $A^{n+1}$ and hence is not normal.

\section{REFERENCES}

1. LEAHY, J. V. and VITULLI, M. A. Seminormal Rings and Weakly Normal Varieties, Nagoya Math. J., 82 (1981), 27-56.

2. SWAN, R. On Seminormality, J.A1g. 67 (1980), 210-229.

3. ZARISKI, 0. and SAMUEL, P. Commutative Algebra, Vo1. I, II, Van Nostrand, New Jersey 1960.

4. ANDERSON, D. F. Seminorma1 Graded Rings, J.P.A.A.A. 21 (1981) 1-8.

5. BREWER, J. W., COSTA, D. L. and MC CRIMMON, K. Seminormality and Root Closure in Polynomial Rings and Algebraic Curves, J. Alg. 58 (1979) 217-226.

6. ANDERSON, D. F. Seminormal Graded Rings II, J.P.A.A.A. 23 (1981) 221-226.

7. BARSHAY, J. Graded Algebras of Powers of Ideals Generated by A-Sequences, J.Alg. 25 (1973) 90-99.

8. MUMFORD, D. Introduction to Algebraic Geometry, Harvard Lecture Notes. 


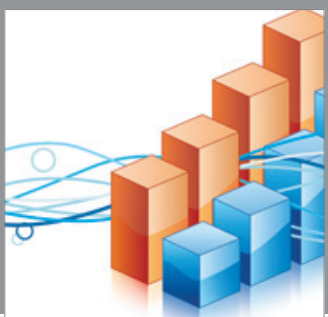

Advances in

Operations Research

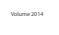

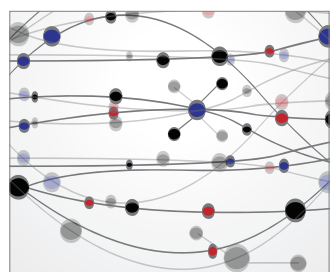

\section{The Scientific} World Journal
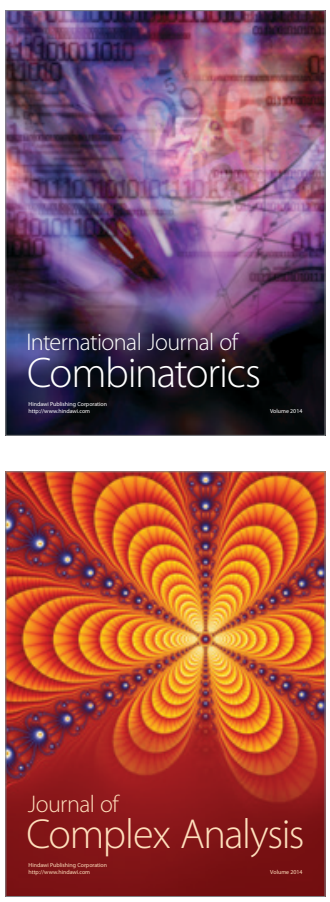

International Journal of

Mathematics and

Mathematical

Sciences
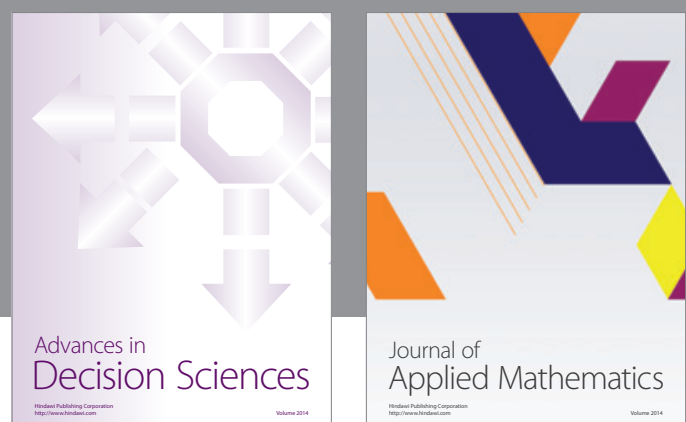

Journal of

Applied Mathematics
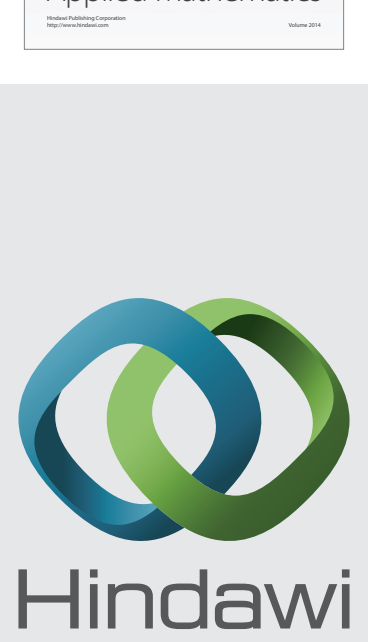

Submit your manuscripts at http://www.hindawi.com
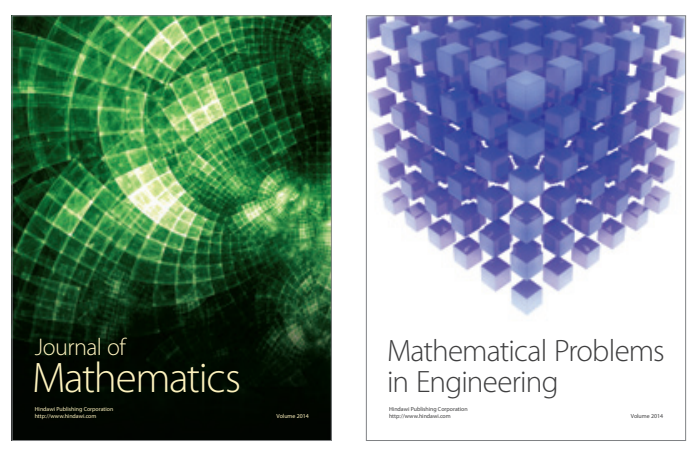

Mathematical Problems in Engineering
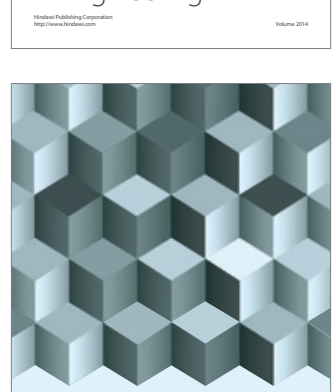

Journal of

Function Spaces
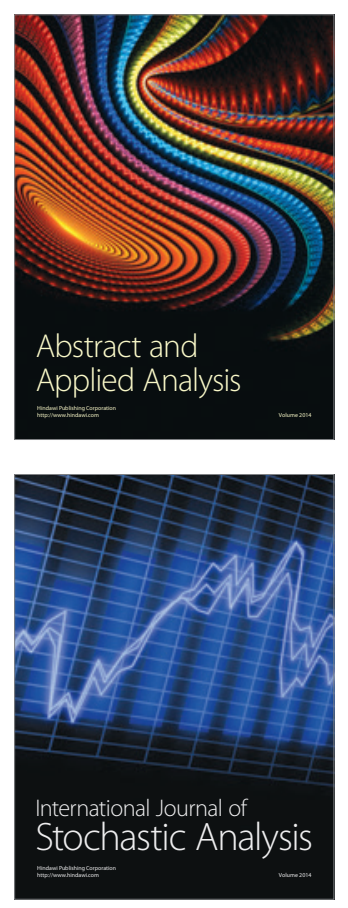

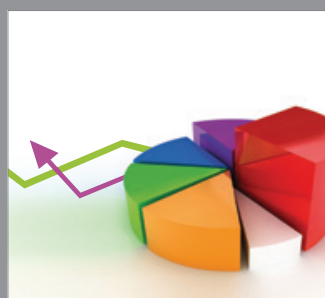

ournal of

Probability and Statistics

Promensencen
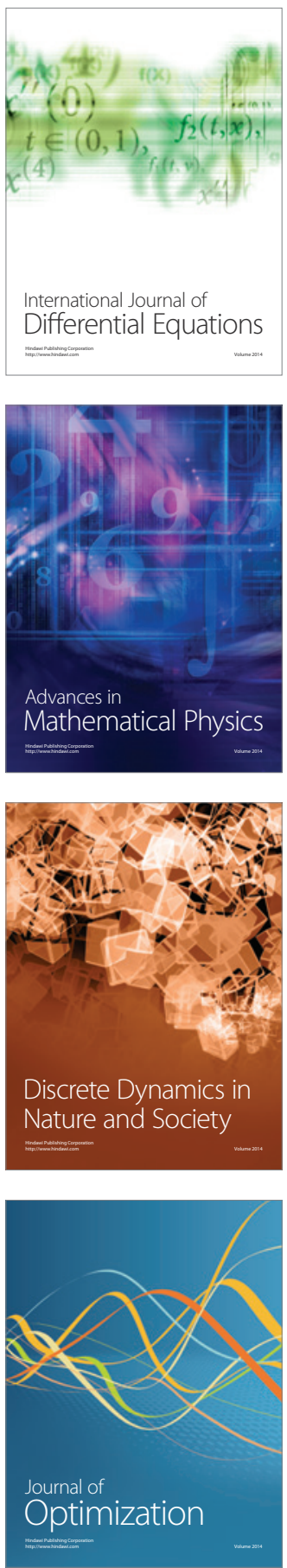